\title{
ASSISTÊNCIA PRÉ-NATAL: COMPETÊNCIAS DESEMPENHADAS POR ENFERMEIROS EM ESFS DO VALE DO SÃO PATRÍCIO-GO.
}

\author{
PRENATAL CARE: SKILLS CARRIED FOR NURSES IN \\ ESFS IN VALE DO PATRÍCIO -GO.
}

\begin{abstract}
Milce Costa
Doutora em Medicina Tropical. Docente da Faculdade de Ceres, Ceres - GO, Brasil.

milcebiomol@yahoo.com.br
\end{abstract}

Camila Regina Pouças Marinho

Enfermeira

camiladoalem@hotmail.com

Ana Paula Pereira Moraes

Enfermeira

moraespereirapaulaana@hotmail.com

Endereço para correspondência: Av. Brasil, S/N, Qd. 13, Morada Verde, Ceres - Go, CEP: 76300-000. Fone/Fax: (62) 3323-1040

\section{RESUMO}

Introdução: Com uma assistência pré-natal adequada, a mortalidade materna que é um grande problema social, poderia ser evitada em $98 \%$ dos casos relacionados ao período gravídico puerperal, já que os coeficientes de mortalidade materna e infantil são influenciados pela qualidade da assistência durante o pré-natal. Metodologia: Trata-se de uma pesquisa de caráter exploratório-descritivo com analise quantitativa, realizada em 13 ESFs dos municípios de Carmo do Rio Verde, Ceres e Uruana em Goiás. A população estudada foram os enfermeiros atuantes nas referidas ESFs, que eram responsáveis pela assistência pré-natal num total de treze enfermeiros. A coleta dos dados foi feita através de aplicação de um questionário fechado. Para analise foram definidos itens imprescindíveis numa consulta de pré-natal como procedimentos relacionados a gestação, ao exame físico geral, ao exame específico gineco-obstétrico e aos exames laboratoriais. Objetivo: Pesquisa com o objetivo de verificar se as ações de pré-natal realizadas pelos enfermeiros das Estratégias Saúde da Família (ESF) dos municípios supracitados desenvolvidas de acordo com o preconizado pelo Ministério da saúde. Resultados e Discussão: Os resultados deste estudo mostram que as competências essenciais esperadas na assistência pré-natal preconizadas pelo Manual "PRÉNATAL E PUERPÉRIO: Atenção Qualificada e Humanizada" do MS foram desenvolvidas pela maioria dos enfermeiros estudados. As ações e procedimentos mais realizados pelos enfermeiros são: A DUM, a DPP, verificação de sinais vitais, determinação do peso e estado 
nutricional, pesquisa de edema, exame de membros inferiores, medida da altura uterina e solicitações de exames laboratoriais (Teste Anti-HIV, Sorologia para Hepatite B (HBsAg), sífilis (VRDL), Rubéola e Toxoplasmose), sendo que 100\% dos enfermeiros estudados afirmaram realizar essas competências. Conclusão: A realização de estudos sobre avaliação de qualidade e relevância do atendimento pré-natal realizado por enfermeiros no estado de Goiás seriam de grande relevância para a consolidação de uma prática bem sucedida.

Palavras-chave: Assistência Pré-natal. Conformidade. Enfermeiro. Gestante.

\section{ABSTRACT}

Introduction: With proper prenatal care, maternal mortality is a major social problem, could be avoided in $98 \%$ of cases related to pregnancy period puerperal, since the maternal and infant mortality rates are influenced by the quality of care during the prenatal period. Metodology: The methodology was a research exploratory, descriptive with quantitative analysis done in 13 health centers. The informations were gotten from a survey done. Then we gave special attention on essencial actions in an office visit as: Exams and investigation corresponding to pregnancy. The answers of the survey were registered as actions of agreement or non agreement with the manual. Objective: The main goal of this research was to verify, if the actions realized by the nurses of Family's Health Program in Carmo do Rio Verde, Ceres and Uruana in Goiás are done according to the manual: Pre Cares on Birth and Puerperium: Qualified and Humanized. Results and discussion: The results of this research show that the main actions related on a manual were done by almost all professionals. The actions that verified 100\% agreement were: "DUM" verification, calculation of "DPP", vital signs, weight determination, edema, nutritional cares, exams that verify all members, measure uterine height, exams to verify communicable diseases. Conclusion: The results of this research show that the assistance given by the nurses to the pregnant ones are according to the Ministry Health Manual.

Key words: Pre Care Birth Assistance, Nurse, Agreement, Pregnant. 


\section{INTRODUÇÃO}

A experiência gestacional é um período muito peculiar na vida da mulher e o nascimento do filho é uma vivência única, portanto, merecem ser assistidos de forma individualizada por profissionais qualificados, pela equipe multidisciplinar, por gestores e pelo governo (VIEIRA et al, 2011)

Visando melhorar a abrangência e a qualidade do pré-natal e das ações realizadas no parto e puerpério, o Ministério da Saúde ao longo de décadas vêm tentando aperfeiçoar os programas existentes de assistência a saúde da mulher. No ano 2000 instituiu o Programa de Humanização de Pré-natal e Nascimento (PHPN) concomitantemente com o lançamento do manual técnico “ASSISTÊNCIA PRÉ-NATAL: Normas e Manuais técnicos”, o qual norteava as ações e capacitava os profissionais a organizar a rede assistencial das práticas de pré-natal (RODRIGUES;NASCIMENTO; ARAÚJO,2011).Já no ano de 2006, levando em consideração os princípios e diretrizes da Política Nacional de Humanização (Humaniza SUS) e as orientações da Organização Mundial de Saúde (OMS), o MS publicou um novo manual técnico denominado: "PRÉ-NATAL E PUERPÉRIO: Atenção Qualificada e Humanizada" trata-se de um manual mais direcionado a assistência humanizada com o propósito de oferecer referência para organização da rede assistencial, qualificação profissional e padronização das práticas de assistência e saúde (BRASIL, 2006).

Com uma assistência pré-natal adequada, a mortalidade materna que é um grande problema social, poderia ser evitada em $98 \%$ dos casos relacionados ao período gravídico puerperal, já que os coeficientes de mortalidade materna e infantil são influenciados pela qualidade da assistência durante o pré-natal (MIRANDA; FERNADES, 2010).A mortalidade materna e neonatal é um grande problema social para o Brasil, no ano de 2003 a Razão da Morte Materna (RMM) foi de 51,74 óbitos por 100.000 nascidos vivos (NV) (BRASIL,2006).

$\mathrm{Na}$ assistência pré-natal são envolvidas várias categorias de profissionais de saúde que buscam alcançar e manter a integralidade da saúde do binômio mãe-filho. O enfermeiro ocupa uma posição de destaque na assistência pré-natal uma vez que é um profissional qualificado para realizar atendimento a gestante, tem grande importância como orientador, pois é ele que trabalha e exerce a função educativa de realizar ações preventivas, de promoção da saúde além de ser agente da humanização (RODRIGUES; NASCIMENTO; ARAÚJO,2011). De acordo com lei do exercício profissional da enfermagem a assistência pré-natal de baixo risco pode ser realizada integralmente pelo profissional enfermeiro. Uma vez que este possui conhecimento teórico-cientifico para prestar esse tipo de assistência as 
gestantes (D.O.U 94.406/87). A consulta de pré-natal realizada pelo enfermeiro é um instrumento de grande relevância, por ter como finalidade a garantia da extensão de cobertura e melhoria da assistência pré-natal, especialmente pelas ações preventivas e promocionais à saúde das gestantes (SHIMIZU; LIMA, 2009).

Um fator importante que marcou o desenvolvimento da assistência pré-natal no Brasil foi a implantação do Programa de Saúde da Família (PSF), hoje denominado Estratégia de Saúde da Família (ESF). A Estratégia é voltada para uma prática universal de saúde, de caráter individual e coletivo. Sendo a principal porta de entrada do sistema e também onde são firmados os vínculos entre a equipe de saúde e comunidade (BRASIL, 2000).

Sabendo-se que teoricamente o profissional enfermeiro possui competência necessária para oferecer essa assistência à gestante e que este é um dos responsáveis pela de consulta pré-natal nas ESFs, o presente estudo pretende verificar se os enfermeiros que respondem pelas ESFs dos municípios de Carmo do Rio Verde, Ceres e Uruana (na região do Vale do São Patrício em Goiás) estão desenvolvendo as ações de assistência pré-natal de acordo com o preconizado pelo Ministério da Saúde. Deste modo, pretende-se identificar e comparar a qualidade do trabalho realizado nesta região por estes profissionais.

\section{METODOLOGIA}

O presente estudo trata-se de uma pesquisa de caráter exploratório-descritivo com análise quantitativa.

\subsection{Local do estudo}

Neste estudo foram avaliadas um total de treze Estratégias da Saúde da Família (ESF). Inicialmente foram enviados ofícios às secretarias municipais de saúde das cidades de Carmo do Rio Verde, Ceres e Uruana, localizadas no vale do São Patrício no Estado de Goiás, solicitando a aprovação da pesquisa nas ESFs das respectivas cidades. Uma vez aprovada, procedeu-se a coleta de dados.

\subsection{População estudada}

A população estudada foram os enfermeiros atuantes nas ESFs citadas, num total de treze enfermeiros. O profissional enfermeiro pesquisado obteve esclarecimento sobre o objetivo da pesquisa e seu anonimato, bem como outros esclarecimentos através do Termo de Consentimento Livre e Esclarecido, o qual foi devidamente assinado pelo profissional que concordou em participar desta pesquisa. Foram excluídos da pesquisa as ESFs nas quais o 
enfermeiro não era o responsável pela assistência pré-natal, os que tiveram mais de uma opção de resposta e os enfermeiros que se recusaram a participar.

2.3 Coleta dos dados

A coleta dos dados foi feita através de aplicação de um questionário fechado, elaborado pelas próprias autoras com base em documentos que traduzem os padrões baseados em evidência científica na prática obstétrica a partir do Manual "PRÉ-NATAL E PUERPÉRIO: Atenção Qualificada e Humanizada" de 2006- Manual Técnico do Ministério da Saúde (MS). Os questionários foram aplicados em horários agendados de acordo com a disponibilidade dos enfermeiros em suas respectivas unidades de saúde.

2.4 Análise dos dados

Para verificar se as das ações de assistência pré-natal realizadas pelos enfermeiros das Estratégias Saúde da Família (ESF) estão sendo desenvolvidas de acordo com o Manual preconizado pelo Ministério da Saúde, priorizamos os itens considerados como práticas indispensáveis numa consulta de pré-natal (MOURA; RODRIGUES, 2002; CUNHA et al, 2009; DUARTE; MAMEDE, 2012) como: a) análise de procedimentos relacionados à gestação; b) realização do exame físico geral; c) realização do exame específico ginecoobstétrico; d) pedidos de exames laboratoriais. A partir dos itens respondidos positivamente (sim) e negativamente (não) registramos as respostas do questionário como ações de conformidade e não conformidade respectivamente com o Manual do MS. As informações obtidas foram registradas no Microsoft Office Excel 2007. Foi utilizada a estatística descritiva para descrição dos dados e após análise, foram feitas tabelas no programa Microsoft Word.

O projeto para o desenvolvimento do presente estudo foi aprovado pelo Comitê de Ética em Pesquisa do Hospital Alberto Rassi - HGG sob o parecer n ${ }^{\circ} 579.264$ 


\section{RESULTADOS E DISCUSSÃO}

Ao realizar este estudo como forma de verificar as ações de pré-natal realizadas pelos enfermeiros das ESFs, deparou-se com a dificuldade de encontrar, na literatura estudos que tratem do mesmo tema no mesmo tipo de instituição. As principais ações realizadas pelos enfermeiros responsáveis pelas ESFs nas cidades de Carmo do Rio Verde, Ceres e Uruana em Goiás encontram-se distribuídas nas tabelas 1, 2, 3 e 4. Em relação à análise de procedimentos relacionados à gestação, o enfermeiro foi questionado dentre outras informações, se durante a consulta de pré-natal analisa: a data da última menstruação (DUM) e a data provável do parto (DPP). As respostas positivas ( $\operatorname{sim}$ ) foram transferidas para as tabelas como conformidades e as respostas negativas (não) como não conformidades em relação às condutas preconizadas pelo manual: "Pré-Natal e Puerpério: Atenção Qualificada e Humanizada" do ano de 2006 do MS. Quanto a DUM e a DPP, 13 (100\%) dos enfermeiros afirmaram analisar estes dados nas consultas de pré-natal. Outros estudos demonstram percentuais um pouco menores quando comparados aos deste estudo em relação à análise e cálculo da DUM $(94,1 \%)$ e DPP $(87,5 \%)$ pelos enfermeiros (CUNHA et al, 2009; MOURA; RODRIGUES, 2002). Deve-se ressaltar que a observação da DUM e da DPP pelos enfermeiros são procedimentos necessários na consulta pré-natal que possibilitam à gestante um melhor planejamento quanto ao parto e puerpério. Além disso, são parâmetros que permitem verificar a possível ocorrência de partos prematuros ou mesmo pós-datismo (MOURA; RODRIGUES, 2002; CUNHA et al, 2009).

Tabela 1: Distribuição e verificação de conformidades e não conformidades segundo MS nas atividades de assistência pré-natal realizadas pelos enfermeiros nas ESFs das cidades de Carmo do Rio Verde, Ceres e Uruana em Goiás.

\begin{tabular}{lcccc} 
& & & \\
Em relação à gestação & Conformidade & Não conformidade \\
\cline { 2 - 4 } & $* N^{\circ}$ & $\%$ & $* N^{\circ}$ & $\%$ \\
\hline DUM & 13 & 100 & - & - \\
DPP & 13 & 100 & - & - \\
Data da percepção dos primeiros movimentos fetais & 10 & 77 & 3 & 23 \\
Sinais e sintomas na gestação em curso & 12 & 92 & 1 & 8 \\
Medicação usada na gestação & 12 & 92 & 1 & 8 \\
Se a gestação foi ou não desejada & 12 & 92 & 1 & 8 \\
\hline *Número de profissionatermeiro & & & &
\end{tabular}

* Número de profissional enfermeiro

Ao verificarmos se os profissionais enfermeiros realizam os exames físicos gerais nas gestantes (Tabela 2), foi possível observar que 13 (100\%) dos enfermeiros pesquisados relataram que fazem o acompanhamento do peso e do estado nutricional da paciente, realizam 
o exame de membros inferiores e pesquisam edema nas consultas. Outro resultado que se destaca nesta competência é o fato de 12 (92\%) dos profissionais não realizarem a palpação da tireóide, sendo portanto, este item categorizado neste estudo como uma não conformidade com o preconizado pelo Ministério da Saúde.

Tabela 2: Distribuição e verificação de conformidades e não conformidades segundo MS nas atividades de assistência pré-natal realizadas pelos enfermeiros nas ESFs das cidades de Carmo do Rio Verde, Ceres e Uruana em Goiás.

\begin{tabular}{lcccc}
\hline Exame físico geral & \multicolumn{2}{c}{ Conformidade } & \multicolumn{2}{c}{ não conformidade } \\
\cline { 2 - 5 } & $* \mathrm{~N}^{\circ}$ & $\%$ & $* \mathrm{~N}^{\circ}$ & $\%$ \\
\hline & 13 & 100 & - & - \\
Verificação de sinais vitais & 1 & 8 & 12 & 92 \\
Palpação da tireoide & 13 & 100 & - & - \\
Determinação do peso e estado nutricional & 12 & 92 & 1 & 8 \\
Inspeção da pele e mucosa & 13 & 100 & - & - \\
Pesquisa de edema & 13 & 100 & - & - \\
Exame MMII & 11 & 85 & 2 & 15 \\
Exame do abdome & & & &
\end{tabular}

Em estudo semelhante realizado por Moura \& Rodrigues (2002), verificou-se que $87,5 \%$ das enfermeiras responsáveis pelas consultas pré-natais avaliaram o estado nutricional das gestantes. A avaliação do estado nutricional destas pacientes é um procedimento de grande importância, pois permite identificar as gestantes com déficit nutricional ou com sobrepeso. Uma vez detectada tal situação, a gestante deverá ser encaminhada pelo enfermeiro para consulta médica. Deve-se destacar que o ganho insuficiente de peso na gestação normalmente está associado a pouca ingestão de alimentos (talvez relacionado ao baixo nível sócio-econômico); ao crescimento intrauterino retardado; à hiperêmese gravídica; às infecções; às parasitoses; às anemias e a outras doenças debilitantes. Quanto ao aumento excessivo de peso na gestação, pode estar associado à macrossomia fetal, ao polidrâmnio, ao edema e à gravidez múltipla (BRASIL, 2000).

Quanto à realização do exame de membros inferiores e pesquisa de edema nas gestantes, Duarte \& Mamede (2012) verificaram que apenas 45,9\% dos enfermeiros realizavam este procedimento. A pesquisa de edema é extremamente necessária para o diagnóstico e monitoramento da Doença Hipertensiva Específica da Gestação (DHEG), também conhecida por pré-eclâmpsia, que pode causar sérias alterações à gestante e ao concepto. A DHEG é uma desordem multissistêmica que pode ocorrer a partir da $20^{\mathrm{a}}$ semana de gestação. É caracterizada por manifestações clínicas associadas, como hipertensão e 
proteinúria, que desaparecem até 12 semanas após o parto (SILVA et al, 2011). A préeclâmpsia/eclâmpsia continua sendo a primeira causa de morte materna no Brasil e determina o maior número de óbitos perinatais, além do aumento significativo do número de neonatos com sequelas, caso sobrevivam aos danos da hipóxia cerebral (BRASIL, 2006). Em virtude de sua estreita associação com quadros de pré-eclâmpsia e eclâmpsia, o edema deve ser bem avaliado na consulta de pré-natal com o objetivo de evitar/amenizar sua possível ocorrência. Embora a prevenção ainda não seja conhecida amplamente, sabe-se que o diagnóstico precoce da DHEG e suporte de saúde qualificado são determinantes de um melhor prognóstico. O atendimento pré-natal e puerperal desempenha importante papel no controle das intercorrências e no cuidado efetivo da DHEG (SILVA et al, 2011).

Em relação ao procedimento de palpação da tireóide, Cunha et al, (2009), observaram que $96,72 \%$ dos enfermeiros que realizavam consultas de pré-natal não realizavam este procedimento. Este procedimento é preconizado pelo MS (para pesquisa de nódulos e outras anormalidades) e o profissional enfermeiro responsável deve estar atento para o aumento fisiológico da glândula da tireóide (geralmente indicando hipertireoidismo). $\mathrm{Na}$ dúvida, o profissional deve solicitar exames ou encaminhar a gestante para o serviço especializado (CUNHA et al, 2009). Quando o hipertireoidismo não é tratado e não regride com a evolução da gestação, algumas complicações maternas e fetais são relatadas. Na gestante podem-se verificar maiores incidências de eclâmpsia, insuficiência cardíaca congestiva, edema agudo de pulmão, arritmias cardíacas e crise tireotóxica. No concepto têm sido relatadas maiores taxas de abortamento, natimortalidade, prematuridade, baixo peso ao nascer e malformações fetais(PINHEIRO et al, 2008).

A Tabela 3 apresenta um panorama das atividades de assistência pré-natal em relação à realização dos exames específicos gineco-obstétricos nas ESFs pesquisadas. Quanto à realização da medida da altura uterina, 13 (100\%) dos profissionais afirmaram fazê-lo durante as consultas. Doze (92\%) dos enfermeiros dizem realizar a ausculta dos BCFe 11 $(85 \%)$, coletam material para realização da colpocitologia entre outros procedimentos.

Tabela 3: Distribuição e verificação de conformidades e não conformidades segundo MS nas atividades de assistência pré-natal realizadas pelos enfermeiros nas ESFs das cidades de Carmo do Rio Verde, Ceres e Uruana em Goiás. 


\begin{tabular}{lcccc}
\hline & & & \\
Quanto ao exame específico, gineco-obstétrico & Conformidade & Não conformidade \\
\cline { 2 - 5 } & $* \mathrm{~N}^{\circ}$ & $\%$ & $* \mathrm{~N}^{\circ}$ & 15 \\
\hline Exame das mamas & 11 & 85 & 2 & - \\
Medida da altura uterina & 13 & 100 & - & 8 \\
Ausculta do BCF & 12 & 92 & 1 & 15 \\
Identificação da situação e apresentação fetal & 11 & 85 & 2 & 39 \\
Inspeção dos genitais internos & 8 & 61 & 5 & 46 \\
Exame especular internos & 7 & 54 & 6 & 15 \\
Exame colpocitológico & 11 & 85 & 2 & 62 \\
Toque vaginal & 5 & 38 & 8 &
\end{tabular}

Neste estudo observamos que a verificação da altura uterina foi realizada por todos os enfermeiros (100\%) durante as consultas de pré-natal. Outros estudos também apresentam altos percentuais de realização deste procedimento, como observado no trabalho de Cunha et al, (2009) (90\%), Koffman \& Bonadio (2005) (97\%) e Benigna et al (2004) (100\%). A verificação da altura uterina no pré-natal é um procedimento recomendado pelo MS em virtude de sua grande importância no monitoramento do crescimento normal do feto e da detecção de suas possíveis alterações. Excluindo-se a possibilidade de erro na realização do procedimento, ao se detectar desvios nos resultados padronizados deve-se encaminhar a gestante para serviços especializados para realização de possíveis intervenções (BRASIL, 2000).

Quanto à realização da ausculta de batimentos cardio-fetais (BCF), 12 (92\%) dos enfermeiros pesquisados neste estudo afirmam realizar este procedimento nas consultas de pré-natal. Em estudo conduzido por Koffman \& Bonadio (2005) verificou-se que este procedimento também foi realizado por $96,7 \%$ dos enfermeiros nas consultas de pré-natal do Amparo Maternal da Escola de Enfermagem da USP-SP. A avaliação deste procedimento é indispensável no pré-natal, uma vez que seu objetivo é constatar a presença, o ritmo, a frequência e a normalidade dos BCF (BRASIL, 2006).

Quanto à coleta de material para realização de colpocitologia, 11 (85\%) dos enfermeiros responsáveis pelas consultas de pré-natal neste estudo, confirmam realizar este procedimento. Comparando-se este resultado com os trabalhos de Duarte \& Mamede (2012) e Gonçalves et al(2009), onde os percentuais de realização deste procedimento são de apenas 
9,4\% e 33,6\% respectivamente, pode-se sugerir que os enfermeiros pesquisados realizam de modo satisfatório tal procedimento que pode revelar o câncer de colo uterino entre outras patologias (AMORIM; MELO, 2009). Acredita-se que os baixos percentuais de coleta colpocitológica encontrados nos estudos supracitados, se devam à controvérsia existente entre os profissionais enfermeiros de que o procedimento de coleta se mal conduzido pode induzir ao aborto (DUARTE; MAMEDE, 2012).

Ao se verificar as solicitações de exames nas consultas de pré-natal (Tabela 4) realizadas pelos enfermeiros responsáveis pelas ESFs pesquisadas, observou-se uma tendência dos enfermeiros em privilegiar alguns dos exames que fazem parte do Programa de Proteção à Gestante - "Teste da mamãe”. A solicitação dos testes: anti-HIV, sorologia para rubéola, toxoplasmose, hepatite B (HBsAG) e sífilis (VDRL) foi realizada por todos os 13 (100\%)enfermeiros pesquisados. No estudo de Cunha et al (2009) observou-se que os enfermeiros também solicitavam amplamente estes exames, sendo que $100 \%$ dos enfermeiros requisitavam anti- HIV, 100\% toxoplasmose, 90\% hepatite B (HBsAG) e 100\% sífilis (VDRL).O acompanhamento dos exames laboratoriais no primeiro e terceiro trimestre gestacional traz amplos benefícios à saúde da gestante e do concepto, uma vez que a maioria das doenças pesquisadas pode ser tratada durante a gravidez reduzindo significativamente os casos de contaminação vertical ou reduzindo as sequelas pelo tratamento precoce do recémnascido (FILHO et al, 2009).

Tabela 4: Distribuição e verificação de conformidades e não conformidades segundo MS nas atividades de assistência pré-natal realizadas pelos enfermeiros nas ESFs das cidades de Carmo do Rio Verde, Ceres e Uruana em Goiás.

\begin{tabular}{lcccc}
\hline \multirow{2}{*}{ Exames laboratoriais solicitados } & \multicolumn{2}{c}{ Conformidade } & \multicolumn{2}{c}{ Não conformidade } \\
\cline { 2 - 5 } & $* \mathrm{~N}^{\circ}$ & $\%$ & $* \mathrm{~N}^{\circ}$ & $\%$ \\
\hline Dosagem de hemoglobina (Hb) & 9 & 69 & 4 & 31 \\
Grupo sanguíneo, fator RH & 11 & 85 & 2 & 15 \\
Sorologia para Hepatite B (HBsAg) & 13 & 100 & - & - \\
Sorologia para sífilis (VRDL) & 13 & 100 & - & - \\
Glicemia em jejum & 11 & 85 & 2 & 15 \\
Sorologia para Rubéola & 13 & 100 & - & - \\
Exame sumário de urina & 11 & 85 & 2 & 15 \\
Exame parasitológico de fezes & 8 & 61 & 5 & 39 \\
Colpocitologia oncótica & 9 & 69 & 4 & 31 \\
Teste Anti-HIV & 13 & 100 & - & - \\
Sorologia para Toxoplasmose & 13 & 100 & - & - \\
\hline * Número de profissional enfermeiro & & & &
\end{tabular}

A Tabela 5 mostra o perfil de capacitação profissional dos enfermeiros responsáveis pelas ESFs das cidades de Carmo do Rio Verde, Ceres e Uruana em Goiás. 
Quanto à formação obstétrica, $0(0 \%)$ nenhum dos enfermeiros afirmou ter este tipo de curso de especialização. No entanto, quando questionados sobre cursos de capacitação para realização de pré-natal, 2 (66,6\%), $3(60 \%)$ e $4(80 \%)$ dos enfermeiros de Carmo do Rio Verde, Ceres e Uruana respectivamente afirmaram ter realizado a referida capacitação. Ao serem questionados sobre o processo de capacitação da equipe de agentes comunitários de saúde que realizam as buscas ativas às gestantes, 13 (100\%) dos profissionais responderam que capacitam seu pessoal.

Do universo pesquisado, nenhum dos enfermeiros possuía especialização em obstetrícia. A mesma situação foi verificada no estudo de Duarte \& Mamede (2011), onde nenhum dos 74 enfermeiros responsáveis por consultas de pré-natal em ESFs e CS (Centros de Saúde) em Cuiabá-MT tinham formação em obstetrícia. Cabe ressaltar que o profissional enfermeiro com formação obstétrica apresenta conhecimento e qualificação complementares que o tornam habilitado para prestar assistência à gestante de forma a garantir a diminuição dos índices de mortalidade materna e perinatal. Dessa forma, é importante investir na formação de especialistas em enfermagem obstétrica para o atendimento à mulher no ciclo gravídico-puerperal, uma vez que a participação dos enfermeiros é de fundamental importância para o fortalecimento da assistência pré-natal na saúde pública (CUNHA et al, 2009). No entanto, em virtude de limitações de recursos sociais e econômicos impostos pela realidade muitas vezes o profissional opta por um curso de capacitação ao invés de realizar a tão desejada especialização.

Tabela 5: Verificação de capacitação dos profissionais enfermeiros responsáveis pelas ESFs de Carmo do Rio Verde, Ceres e Uruana em Goiás.

\begin{tabular}{|c|c|c|c|c|c|c|}
\hline \multirow[t]{2}{*}{ Capacitação profissional } & \multicolumn{2}{|c|}{$* * \mathrm{CRV}=3$} & \multicolumn{2}{|c|}{ Ceres $=5$} & \multicolumn{2}{|c|}{ Uruana $=5$} \\
\hline & $* \mathrm{~N}^{\circ}$ & $\%$ & $* \mathrm{~N}^{\circ}$ & $\%$ & $* \mathrm{~N}^{\circ}$ & $\%$ \\
\hline Possui especialização em obstetrícia & 0 & - & 0 & - & 0 & - \\
\hline $\begin{array}{l}\text { Possui curso de capacitação para realização } \\
\text { de pré-natal }\end{array}$ & 2 & 33,3 & 3 & 60 & 4 & 80 \\
\hline $\begin{array}{l}\text { Capacita a equipe de agentes comunitários } \\
\text { de saúde para visitas as gestantes }\end{array}$ & 3 & 100 & 5 & 100 & 5 & 100 \\
\hline
\end{tabular}


Quanto aos enfermeiros que afirmaram ter realizado cursos de capacitação em assistência pré-natal, pudemos observar um percentual satisfatório de profissionais que afirmaram tê-lo feito,66,6\%, 60\% e 80\% nas ESFs em Carmo do Rio Verde, Ceres e Uruana respectivamente. No estudo de Cunha et al (2009) conduzido em ESFs em Rio BrancoAC, $88,24 \%$ dos enfermeiros pesquisados relatam ter feito capacitação para assistência prénatal. Os cursos de capacitação em assistência pré-natal qualificam o profissional de forma a contribuir para uma assistência de qualidade com base nas ações recomendadas tanto pelas Políticas Públicas de Saúde quanto pelo Programa Nacional de Humanização do Parto e Nascimento (SÃO PAULO, 2009).

Em relação à capacitação da equipe de agentes comunitários de saúde (ACS), 100\% dos enfermeiros responsáveis pelas ESFs responderam que capacitam seu pessoal. Uma vez que a capacitação dos ACS promove a busca ativa precoce por gestantes, pode-se sugerir que as gestantes das referidas comunidades assistidas pelas ESFs geralmente começam a receber assistência pré-natal dentro do tempo preconizado pelo MS, que é de realizar a primeira consulta de pré-natal nos primeiros 120 dias de gestação (BRASIL, 2006).

Os resultados deste estudo revelam que as competências essenciais esperadas na assistência pré-natal pelos enfermeiros das ESFs estudadas, foram desenvolvidas satisfatoriamente de acordo com o preconizado pelo Manual do MS, entretanto, algumas foram realizadas com baixa frequência, ou seja, não foram realizadas em todas as consultas, indicando a necessidade de esclarecimentos aos enfermeiros sobre a importância da incorporação de protocolos assistenciais e qualificação dos enfermeiros através de cursos de especialização (CUNHA et al., 2009). 


\section{CONCLUSÃO}

Foram inúmeras as competências identificadas na assistência pré-natal realizadas pelos enfermeiros responsáveis pelas ESFs nas cidades de Carmo do Rio Verde, Ceres e Uruana em Goiás. No entanto, as principais ações discutidas e realizadas pelos profissionais neste estudo foram os itens considerados como práticas indispensáveis numa consulta de pré-natal como: procedimentos relacionados à gestação; realização do exame físico geral; realização do exame específico gineco-obstétrico e pedidos de exames laboratoriais.

De modo geral, a maioria dos resultados deste estudo revela que as ações essenciais esperadas na assistência pré-natal, preconizadas pelo Manual: "PRÉ-NATAL E PUERPÉRIO: Atenção Qualificada e Humanizada" do MS, foram desenvolvidas satisfatoriamente pelos enfermeiros das ESFs pesquisadas, levando a crer que tais resultados possam ser entendidos como um indício da aplicação das orientações estabelecidas pelo Ministério da Saúde. Entretanto, algumas ações não foram realizadas em todas as consultas, indicando a necessidade de esclarecer os enfermeiros sobre a importância da incorporação dos protocolos preconizados na assistência pré-natal.

Do universo pesquisado, nenhum dos enfermeiros possuía especialização em obstetrícia. No entanto, pode-se observar um percentual satisfatório de profissionais que afirmaram ter realizado cursos de capacitação em assistência pré-natal,66,6\%, 60\% e 80\% nas ESF sem Carmo do Rio Verde, Ceres e Uruana respectivamente. Cabe ressaltar que o profissional enfermeiro com formação obstétrica apresenta conhecimento e qualificação complementares que o tornam habilitado para prestar assistência à gestante de forma a garantir a diminuição dos índices de mortalidade materna e perinatal. Este resultado sugere a necessidade de expor aos enfermeiros a real importância de se investir na sua qualificação através de cursos de especialização. 


\section{AGRADECIMENTOS}

Aos enfermeiros que aceitaram participar desse estudo, sem eles não seria possível a realização do mesmo. E as secretarias de saúde dos municípios de Carmo do Rio Verde, Ceres e Uruana por apoiar a nossa pesquisa. 


\section{REFERÊNCIAS BIBLIOGRÁFICAS}

AMORIM, M. M. R; MELO, A. S. O. Avaliação dos exames na rotina no pré-natal (Parte 1). Rev. Bras. Ginecol. Obstet. [s. 1.] v. 31 n. 3 p. 148-55, 2009.

BENIGNA, M. J. C; NASCIMENTO, W. G; MARTINS, J. L. Pré-natal no Programa Saúde da Família (PSF): Com a Palavra, os Enfermeiros. CogitareEnferm. [s. 1.] v.9 n.2 p.23-31, jun/dez, 2004.

BRASIL. Decreto $\mathrm{n}^{\circ}$ 94406, de 08 de junho de 1987. Dispõe sobre o exercício da Enfermagem, e da outras providencias. Publicado no D.O.U. Seção I- fls. 8.853 a 8.855. 1987.

BRASIL. Ministério da Saúde. Secretária de Atenção à Saúde. Pré-natal e Puerpério: atenção qualificada e humanizada: manual técnico. Brasília; 2006. 163 p.

BRASIL. Ministério da Saúde. Secretária de Políticas de Saúde. Assistência Pré-natal: normas e manuais técnicos. Brasília; 2000. 56 p.

CUNHA, M. A; DOTTO, L. M. G; MAMEDE, M. V; MAMEDE, F. V. Assistência pré-natal: competências essenciais desempenhadas por enfermeiros. Esc Anna Nery Ver Enferm. [s.1.] v.13 n.1 p.146-156 2009.

DUARTE, S. H. D; MAMEDE, V. M. Estudo das competências essenciais na atenção prénatal: ações da equipe de enfermagem em Cuiabá, MT. Enfermagem em Foco. [s. 1.], v.3 n. 2 p. 80-75, 2012.

FILHO, C. G; FILHO, J. V.M; GOMES, M. M. LUQUETTI, A. O. Triagem pré-natal ampliada: Teste da mamãe. Vita et Sanitas. Trindade, n.3, jan/dez, 2009.

GONÇALVES, C. V; COSTA, J. S. D; DUARTE, G; MARCOLIN, A. C; LIMA, L. C. V; GARLET, G; BIANCHI, M. S; SAKAI, A. F. Avaliação da frequência de realização do exame físico das mamas, da colpocitologia cervical e da ultrassonografia obstétrica durante a 
assistência pré-natal. Uma inversão de valores. Rev. Assoc. Med. Bras. [s. 1.]v.55 n. 3 p.209$5,2009$.

KOFFMAN, M. D; BONADIO, I.C. Avaliação da atenção pré-natal em uma instituição filantrópica da cidade de São Paulo. Rev. Bras. Saúde Matern. Infant. Recife, v.5 n.1 p. $32-$ 23, dez, 2005.

MIRANDA, F. J. S; FERNANDES, R.A.Q. Assistência Pré-Natal: Estudo de Três Indicadores. Rev. Enferm. URJ. Rio de Janeiro, v.18 n. 2 p. 179-84, abr/jun, 2010.

MOURA, E. R. F; RODRIGUES, M. S. P. Desempenho profissional de enfermeiras na assistência pré-natal. Rev. RENE. Fortaleza, v.3 n.1 p. 27-33 jan/jun, 2002.

PINHEIRO, A. T; COSTA, R. A. A; ABBADE, J. F; MAGALHÃES, C. G; MAZETO, G. M. F.S. Hipertireoidismo na gravidez: repercussões materno-fetais. Rev. Bras. Ginecol. Obst. Botucatu, v.30 n. 9 p. 452-08, 2008.

RODRIGUES, E. M; NASCIMENTO, R. G; ARAÚJO, A. Protocolo na assistência pré-natal: ações facilidades e dificuldades dos enfermeiros da estratégia saúde da família. Ver. Esc. Enferm. USP. [S.L]. v. 45 n. 5 p.1041-1047, fev, 2011.

SÃO PAULO. Secretaria Municipal de Saúde. Coordenadoria Regional de Saúde Leste; RH/Desenvolvimento; 2009. 8 p.

SHIMIZU, H. E; LIMA, M. G. As dimensões do cuidado pré-natal na consulta de enfermagem. Rev. Bras. Enferm. Brasília, v.62 n. 3 p. 387-92, maio-jun, 2009.

SILVA, E. F; CORDOVA, F. P; CHACHAMOVICH, J. L. R; ZÁCHIA, S. A. Percepções de um grupo de mulheres sobre a doença hipertensiva específica da gestação. Ver. Gaúcha Enferm. Porto Alegre, v. 32 n. 2 p. 316-22, jun, 2011. 
VIEIRA, S. M; BOCK, L. F; ZOCCHE, D. A; PESSOTA, C. U. Percepção das puérperas sobre a assistência prestada pela equipe de saúde no pré-natal. Texto Contexto Enferm. Florianópolis, v.20 p. 255-6, 2011. 